\title{
Determinantes do índice de massa corporal em adolescentes de escolas públicas de Piracicaba, São Paulo
}

\author{
Determining of body mass index \\ in adolescents from public schools in Piracicaba, São Paulo State
}

\author{
Alexandre Romero ${ }^{1}$ \\ Betzabeth Slater ${ }^{1}$ \\ Alex Antonio Florindo ${ }^{2}$ \\ M aria do Rosário Dias de Oliveira Latorre ${ }^{3}$ \\ Cláudia Cezar ${ }^{4}$ \\ M arina Vieira da Silva ${ }^{5}$
}

${ }^{1}$ Departamento de Nutrição, FaculdadedeSaúdePública, Universidade de São Paulo. Av. Dr.Arnaldo 715,

Cerqueira Cesar. 01246-904 São Paulo SP. aleromero@usp.br

2 Escola de Ciências, Artese Humanidades,

Universidade deSão Paulo. ${ }^{3}$ Departamento de Epidemiologia, Faculdade deSaúdePública,

Universidade de São Paulo.

${ }^{4}$ Instituto Perfil Esportivo.

${ }^{5}$ Departamento de

Agroindústria, Alimentose Nutrição, Escola Superior deAgricultura Luiz de Queiroz, Universidade de São Paulo.
Abstract The objective of this study was to assess determining factors of body mass index (BMI) in adolescents enrolled in public schools in Piracicaba, São Paulo. The sample had 328 adolescents from both genders; minimum age was 10 years old. We have assessed weight, height, sexual maturity, physical activity, and diet. A multiple linear regression model wasused to assess the association between independent variables and BMI. While girls considered physically active presented greater mean BMI than those insufficiently active, boys did not present statistical difference between mean BMI among those active and those insufficiently active. Sexual maturity was a determinant for BMI, for both genders, reinforcing the idea that it is essential to take this variable into account in studies that assess the nutritional situation in adolescents. Webelievethat the methods used in the present study, which are normally used in similar surveys, have important limitations to assess the influence of variables such as the level of physical activity and food intake on the BMI of adolescents. Thus, it is important to improve these methods and adopt them in further studies.

Key words Adolescents, Body mass index, Physical activity, Food intake
Resumo 0 objetivo do presente estudo foi verificar os fatores determinantes do índice de massa corporal (IM C) de adolescentes matriculados nas escolas públicas de Piracicaba, São Paulo. A amostra foi constituída por 328 adolescentes de ambos os sexos, com idade mínima de dez anos. Verificou-sepeso, estatura, maturação sexual, ati vidade física e consumo alimentar. Foi usado um modelo de regressão linear múltipla para verificar a associação entre as variáveis independentes e o IM C. Enquanto as meninas consideradas fisicamente ativas apresentaram maior média de IMC do que as insuficientemente ativas, a média do IM C dos meninosnão apresentou diferença estatística quando comparada entre meninos ativos e insuficientemente ativos. A maturação sexual foi determinante do IM C, para ambos os sexos, reforçando a ideia de que é fundamental levar em consideração essa variável em estudos que avaliam o estado nutricional em adolescentes. Acredita-se que os mé todos utilizados no presente estudo, os quais são normalmente utilizados em pesquisas semelhantes, apresentaram importantes limitações para avaliar a influência do nível de atividade física e do consumo alimentar sobre o IM C dos adolescentes. Dessa forma, ressalta-se a necessidade de aprimoramento desses métodos para adoção em futuros estudos.

Palavras-chave Adolescentes, Índice de massa corporal, Atividade física, Consumo alimentar 
Introdução

$\mathrm{Na}$ adolescência, fase que se estende dos dez aos dezenove anos ${ }^{1}$, verifica-se uma expressiva variação na composição corporal. Isto acontece porque o crescimento é um processo dinâmico e complexo, e pode ser influenciado por vários fatores, como her editariedade, ingestão de alimentos, atividade física, idade, sexo, os quais interferem sobre o tamanho e a forma do indivíduo?

Por meio da avaliação das dimensões corporais, pode-se verificar o estado de saúde dos indivíduos. Como exemplo, pode-se citar o índice de massa corporal (IMC), índice amplamente utilizado na avaliação do estado nutricional ${ }^{3}$.

A Organização M undial da Saúde ${ }^{1}$ recomenda a utilização do IM C para diagnosticar tanto a obesidade quanto a desnutrição em adolescentes. São vários os autores que recomendam eutilizam o IM C para avaliar o estado nutricional de joven $\mathrm{S}^{4,5}$.

O IM C é bastante utilizado, principalmente em estudos populacionais, devido ao baixo custo, à simplicidade para realização das medidas e à alta reprodutibilidade.

Vários fatores podem influenciar o IMC, entre eles a atividade física. A redução da atividade física por um longo período pode levar a um ganho de peso corpóreo ${ }^{3}$ e, por consequência, ao aumento do IM C. Por outro lado, o aumento da atividade física vigorosa pode reduzir a chance dos adolescentes apresentarem valores de IMC acima dos valores adequados para a saúde ${ }^{6}$.

Outro fator que pode influenciar o IM C é a al imentação, pois enquanto 0 aumento da ingestão de energia, acima das necessidades orgânicas, por um período prolongado pode levar ao ganho do peso corpóreo ${ }^{3}$, a ingestão energética abaixo das necessidades orgânicas provoca uma perda de peso progressiva ${ }^{7}$, levando à redução do IMC.

A maturação sexual que ocorre durante a adolescência pode provocar aumentos significativos nas massas óssea, muscular e gorda ${ }^{8}$, tornando a maturação sexual um fator importante para o aumento do IMC.

I dentificar os fatores determinantes do IMC, em adolescentes, é imprescindível para elaboração de estratégias que tenham como propósito prevenir e tratar distúrbios nutricionais ainda na juventude. Dessa forma, o presente estudo teve como objetivo identificar os fatores determinantes do índice de massa corporal de adolescentes matriculados nas escolas públicas de Piracicaba, São Paulo.

\section{Métodos}

Este trabalho vincula-se à pesquisa intitulada "Consumo dietético e atividade física como determinantes das mudanças do índice de massa corporal de uma coorte de adolescentes matriculados na rede pública de ensino da cidade de Piracicaba, São Paulo", financiada pela FAPESP (Processo 02/9521-9).

Trata-se de um estudo transversal no qual foram selecionados 392 adolescentes de ambos os sexos, com idade mínima de dez anos, alunos matriculados nas 4a, 5 a, 6 a ou 7 a séries das escolas públicas de Piracicaba.

0 procedimento de amostragem foi do tipo aleatório simples, estratificado por séries ( $1^{\mathrm{a}}$ a $4^{a}$ a série e $5^{\mathrm{a}}$ a $8^{\mathrm{a}}$ série), sendo realizado em duas etapas. Na primeira etapa, as unidades primárias de amostragem (UPA), que correspondem às escolas do município, foram ordenadas segundo as regiões. Foram sorteadas onze escolas, considerando que dez situavam-se em áreas urbanas e uma, na área rural.

$\mathrm{Na}$ segunda etapa da amostragem, foram ordenadas as unidades secundárias de amostragem, que correspondem às classes (séries) das escolas. Em cada UPA, sortearam-se as classes por amostragem aleatória simples novamente. De cada classe selecionada, realizou-se um sorteio de determinado número de alunos, dependendo do porte da escola.

Em novembro de 2004, os participantes foram submetidos à aferição de medidas antropométricas, avaliação do estágio de maturação sexual e entrevistas por meio de questionário, possibilitando a coleta de informações sobre estatura, peso, maturação sexual, idade, sexo, consumo de energia e nível de atividade física. Para tanto, oito pesquisadores de campo receberam treinamento específico.

Para avaliação do peso corporal, os adolescentes utilizaram roupas leves e estavam descalços. Foram posicionados sobre uma balança eletrônica do tipo plataforma, da marca Tanita $\bigotimes_{\text {, }}$ com capacidade para $150 \mathrm{~kg}$ e sensibilidade de 100 gramas. Para a aferição da estatura, foi utilizado um estadiômetro da marca Seca®, com escala em milímetros. 0 mesmo foi fixado em um suporte de madeira, confeccionado para o estudo com a finalidade de manter um ângulo reto entreo piso ea parede. N esta medição, os indivíduos mantiveram os pés juntos, calcanhares encostados na parede, em postura ereta, com ol har fixo no horizonte. Tanto o peso quanto a estatura foram aferidos em duplicata, sendo adotado 
o valor médio das duas medidas para o cálculo do índice de massa corporal (IMC).

A avaliação da maturação sexual foi realizada por meio da auto-avaliação e utilizou planiIhas demonstrativas dos estágios de Tanner em desenhos. Tendo em vista que o presente estudo incluiu participantes na faseinicial da adolescência, adotou-se a classificação proposta pela Organização Mundial da Saúde ${ }^{1}$ para agrupar os participantes entre pré-púberes e púberes. Para tanto, foram utilizados somente os dados relativos ao desenvolvimento de mamas no sexo feminino e da genitália no sexo masculino.

O nível de atividade física foi avaliado por meio da aplicação do Questionário de Atividade Física para Adolescentes (QAFA), o qual foi elaborado evalidado por Florindo et al. ${ }^{9}$, sendo que a validação foi realizada com adolescentes pertencentes à população desse estudo. O QAFA é composto por dezessete questões divididas em dois blocos: 1) esportes e exercíciosfísicos (quinzequestões), e2) atividades físicas de locomoção (duas questões). 0 questionário avalia a atividade física semanal e anual efoi padronizado para gerar um escore final em minutos (semanal e anual). 0 resultado do escore foi utilizado como variável contínua (minutos de atividade física) e como variável dicotômica, categorizada em insuficientemente ativo eativo, tendo como ponto de corte trezentos minutos semanais de atividades físicas ${ }^{10}$.

É oportuno salientar que a educação física escolar não está incluída no QAFA, pois se sabe que as atividades realizadas durante as aulas de educação física não têm como objetivo melhorar 0 nível de aptidão física, mas sim sensibilizar e orientar sobre a prática de exercícios físicos regulares, os quais poderão ser praticados fora da escola. Sendo assim, as aulas são usualmente compostas por atividades de baixa intensidade e não influenciam significativamente a aptidão física.

$O$ consumo de energia foi avaliado por meio da aplicação do Q uestionário de Frequência Alimentar para Adolescentes (QFAA), validado por Slater et al. ${ }^{11}$. O QFAA foi reestruturado eaplicado em estudo-piloto realizado com dezesseisadolescentes voluntários de escolas públicas dePiracicaba. Constatou-se a necessidade de realizar alterações em sua estrutura, como inclusão de preparação de alimentos típicos da região e exclusão do item sucos não-adoçados devido a sua baixa frequência de consumo.

Conforme recomendação de Andrade et al. ${ }^{5}$, 52 adolescentes foram excluídos por apresentarem um consumo de energia inferior a $500 \mathrm{kcal}$ ou superior a $6.000 \mathrm{kcal}$. Também foram excluídos doze indivíduos cujos questionários e fichas de avaliação estavam incompletos. Dessa forma, a amostra final foi constituída por 328 adolescentes $(83,7 \%$ da amostra inicial).

Para análise descritiva, foram utilizadas medidas de tendência central e de dispersão.

0 teste de Kolmogorov-Smirnov foi utilizado para avaliar a aderência dos valores das variáveis quantitativas contínuas à distribuição normal. A partir dos resultados deste teste, foi possível decidir se seriam utilizados os testes paramétricos ou não-paramétricos. Foram comparadas as médias de idade, IM C, minutos de atividadefísica e consumo deenergia entremeninose meninas usando o teste de Mann Whitney. Para comparação da maturação sexual segundo sexo, foi utilizado o teste qui-quadrado.

Com o objetivo de realizar comparações entre as médias de IMC de adolescentes pré púberese púberes eentreindivíduosinsuficientemente ativos e ativos, foi utilizado o teste de Mann Whitney, por se tratar de duas amostras independentes.

Para comparar as médias de IM C obtidas para os escolares integrantes de distintos estratos etários, foi utilizado o testedeKruskal Wallis. Por meio do teste Tukey (HSD), foram estabelecidas comparações múltiplas para verificar possível diferença estatisticamentesignificativa entre três ou mais médias. Esses testes não- paramétricos foram utilizados devido ao fato de a variável IMC não apresentar distribuição normal.

Para verificar a relação entre a variável dependente IM C e as variáveis independentes do estudo, foi utilizado o coeficiente de correlação de Spearman. Foram construídos dois modelos de regressão linear múltipla: um para os meninos e outro para as meninas.

As variáveis que apresentaram correlação estatisticamente significativa $(p<0,05)$, ou que tinham reconhecida relevância epidemiológica no contexto do trabalho, foram selecionadas para o modelo de análise de regressão linear múltipla, no qual poderia ser verificada a existência de associação entreo IM C ecada variável selecionada.

Após o processo de modelagem, observouse que os resíduos estão adequados e os modelos não apresentam viés.

Para todas as análises estatísticas de interesse, estabeleceu-seum nível designificância de 5\%. A análise estatística do estudo foi realizada com o auxílio do programa de computador Statistical Package for the Social Sciences (SPSS, 2000), versão 13.0 . 
Este projeto foi aprovado pelo comitê de Ética da Faculdade de Saúde Pública da USP com protocolo de pesquisa $n-1340$. Participaram do estudo apenas os adolescentes que apresentaram o termo de consentimento livre e esclarecido assinado pelos pais ou responsáveis.

\section{Resultados}

Integram as análises dados relativos a 150 meninos ( $45,7 \%$ da amostra total) e 178 meninas (54,3\%).

Do total da amostra, $54,9 \%$ eram insuficientemente ativos ( $<300 \mathrm{~min} / \mathrm{sem})$, sendo $65 \%$ do sexo feminino. No que se refere à maturação se xual, a amostra apresentou $21,3 \%$ de pré-púberes, dos quais $72,9 \%$ eram do sexo masculino. A variável maturação sexual apresentou diferença estatisticamente significativa na proporção de adolescentes pré-púberes entre meninos e meninas, respectivamente $34 \%$ e 10,7\% ( $p<0,001)$.

A Tabela 1 mostra a estatística descritiva das variáveis quantitativas. N essa tabela, observa-se que o tempo de atividade física apresenta uma diferença estatisticamente significativa, a qual demonstra que os meninos apresentam um tempo em minutos de atividade física maior do que o tempo apresentado pelas meninas.

No presente estudo, optou-se por fazer a análise em separado por sexo devido às diferenças queforam encontradas entremeninos emeninas no que se refere ao nível de atividade física e a maturação sexual. Estas diferenças são importantes, uma vez que o nível de atividade física ea maturação sexual podem ser possíveis determinantes do IMC dos adolescentes em estudo.

$\mathrm{Na}$ Tabela 2, apresenta-se a variável IM C se gundo estágio puberal, nível de atividade física e idade dos adolescentes de ambos os sexos.
Observa-se uma diferença estatisticamente significativa entre a média de IMC obtida para os prépúberes e púberes, sendo o valor maior para os púberes.

Em relação ao nível de atividade física, constata-se uma diferença estatisticamente significativa apenas para as meninas, sendo a média de IM C maior entre as adolescentes consideradas ativas fisicamente. No que se refere às médias de IMC, de acordo com as diferentes categorias de idade, verifica-se que a média do IM C das meninas de catorze equinze anos de idade ésignificativamente maior do que a média de IMC das adolescentes de dez anos. Já para os meninos não foi identificada diferença estatística entre as mé dias de IM C, nas diferentes categorias de idade.

A Tabela 3 apresenta resultados da correlação entreo IM C eas variáveisidade, consumo de energia etempo de atividade física. N ota-se que, à medida queaumenta a idadedas meninas, cresce - IM C, pois se verifica, para esta variável, uma correlação positiva e significativa, apesar de fra$\mathrm{ca}(r=0,31 ; p<0,001)$. 0 mesmo tipo de correlação é observado para tempo de atividade física $(r=0,22 ; p=0,003 ;)$.

Para os meninos, a única variável que foi estatisticamente relacionada ao IM C foi a maturação sexual. Para verificar se esta associação éindependente deidade, consumo de energia etempo de atividadefísica, foi feita a análise de regressão linear múltipla apresentada na Tabela 4. Nessa tabela, observa-se que, para os meninos, independente das outras variáveis citadas, o pré púbere tem, em média, - 1,336 ( $p=0,050)$ de IMC, quando comparado com o púbere.

Para as meninas, foram selecionadas para a modelagem múltipla as variáveis idade, maturação sexual, consumo de energia etempo de atividade física (Tabela 5). Verifica-se que foram determinantes independentes a idade e a matura-

Tabela 1. Estatística descritiva das variáveis demográficas, antropométricas, dietéticas e de atividade física de adolescentes de Piracicaba, segundo sexo. São Paulo, 2004.

\begin{tabular}{|c|c|c|c|c|c|}
\hline \multirow{2}{*}{ Variáveis } & \multicolumn{2}{|c|}{ Masculino $n=150$} & \multicolumn{2}{|c|}{ Feminino $n=178$} & \multirow{2}{*}{ Valor de $p^{\text {a }}$} \\
\hline & M édia ( $d p)$ & Mínimo-máximo & Média (dp) & Mínimo-máximo & \\
\hline I dade (anos) & $12,4(1,5)$ & $10,1-15,8$ & $12,2(1,5)$ & $10-15,3$ & 0,113 \\
\hline IMC & $19,8(3,9)$ & $13,3-32,2$ & $19,4(3,7)$ & $13,0-36,6$ & 0,411 \\
\hline Tempo de AF (minutos/semana) & $493,0(513,1)$ & $0,0-2715,0$ & $285,5(330,1)$ & $0,0-2040,0$ & $<0,001$ \\
\hline Consumo de energia (kcal/dia) & $3408,7(1108,6)$ & $1257,2-5903,5$ & $3405,3(1173,0)$ & $917,1-5933,9$ & 0,963 \\
\hline
\end{tabular}

TestedeM ann-Whitney.

AF - Atividadefísica. 
ção sexual. 0 tempo de atividade física e o consumo de energia perderam sua significância na presença das outras variáveis e optou-se por deixá-las como variáveis de ajuste.

Para cada ano de idade nas meninas, há um aumento médio de 0,463 no IM C ( $p=0,014)$ independente das outras variáveis. Observa-se, também, que as adolescentes pré púberes têm, em média, - 2,197 ( $p=0,016)$ de IM C, quando comparadas com as adolescentes púberes.

Tabela 2. Comparação das médias do índice de massa corporal dos adolescentes de Piracicaba, segundo variáveis de interesse. São Paulo, 2004.

\begin{tabular}{|c|c|c|c|c|c|c|}
\hline \multirow{2}{*}{ Variáveis } & \multicolumn{3}{|c|}{ Meninos } & \multicolumn{3}{|c|}{ Meninas } \\
\hline & $\mathrm{N}$ & $\begin{array}{c}\text { IMC } \\
\text { Média (DP) }\end{array}$ & $P$ & $\mathrm{~N}$ & $\begin{array}{c}\text { IMC } \\
\text { M édia (DP) }\end{array}$ & $p$ \\
\hline \multicolumn{7}{|l|}{ M aturação sexual } \\
\hline Pré-púbere & 51 & $18,9(4,2)$ & $0,003^{b}$ & 19 & $16,7(2,3)$ & $<0,001^{b}$ \\
\hline Púbere & 99 & $20,2(3,7)$ & & 159 & $19,6(3,7)$ & \\
\hline \multicolumn{7}{|l|}{ Nível de atividade física } \\
\hline Insuficientemente ativo & 63 & $19,3(3,6)$ & $0,345^{b}$ & 117 & $18,8(3,7)$ & $0,003^{b}$ \\
\hline Ativo & 87 & $20,1(4,1)$ & & 61 & $20,3(3,6)$ & \\
\hline \multicolumn{7}{|l|}{ Idade } \\
\hline 10 & 33 & $19,3(3,9)$ & $0,567^{c}$ & 60 & $18,2(3,5)^{*}$ & $0,001^{c}$ \\
\hline 11 & 30 & $20,9(5,3)$ & & 30 & $19,3(4,2)$ & \\
\hline 12 & 32 & $18,9(2,8)$ & & 19 & $19,2(2,8)$ & \\
\hline 13 & 24 & $19,9(4,0)$ & & 41 & $20,1(4,2)$ & \\
\hline $14+15$ & 31 & $19,9(3,1)$ & & 28 & $20,8(2,6)^{*}$ & \\
\hline
\end{tabular}

${ }^{\mathrm{b}}$ TestedeM ann Whitney.

' Teste de Kruskal-Wallis.

*Diferença estatisticamente significativa.

Tabela 3. Coeficiente de correlação de Spearman entre o IMC e as variáveis idade, tempo de atividade física e consumo de energia dos adolescentes de Piracicaba. São Paulo, 2004.

\begin{tabular}{lccccc}
\hline \multirow{2}{*}{ Variáveis descritivas } & \multicolumn{3}{c}{ Índice de massa corporal } \\
\cline { 2 - 3 } & \multicolumn{2}{c}{ Meninos } & & \multicolumn{3}{c}{ Meninas } \\
\cline { 2 - 3 } \cline { 5 - 6 } & $r^{a}$ & $p$ & & $r^{a}$ & $p$ \\
\hline Idade (anos) & 0,09 & 0,281 & & 0,31 & $<0,001$ \\
Tempo de AF (minutos/semana) & 0,09 & 0,281 & & 0,22 & 0,003 \\
Consumo de energia (kcal/dia) & $-0,12$ & 0,160 & & $-0,04$ & 0,568 \\
\hline
\end{tabular}

${ }^{a}$ Coeficiente de Correlação deSpearman.

AF - Atividadefísica.

Tabela 4. Análise de regressão linear múltipla do IMC para os adolescentes do sexo masculino de Piracicaba. São Paulo, 2004.

\begin{tabular}{lcc}
\hline \multicolumn{1}{c}{ Variáveis } & Coeficiente de regressão & Valor de $p$ \\
\hline M aturação sexual (pré-púbere) & $-1,336$ & 0,050 \\
Tempo de AF (minutos/semana) & 0,001 & 0,346 \\
Consumo de energia (kcal/dia) & $-0,001$ & 0,057 \\
\hline
\end{tabular}

$r^{2}$ ajustado para os meninos $=0,04$.

AF - Atividadefísica. 
Tabela 5. Análise de regressão linear múltipla do IMC para os adolescentes do sexo feminino de Piracicaba. São Paulo, 2004.

\begin{tabular}{lcc}
\hline \multicolumn{1}{c}{ Variáveis } & Coeficiente de regressão & Valor de $p$ \\
\hline Maturação sexual (pré-púbere) & $-2,197$ & 0,016 \\
Tempo de AF (minutos/semana) & 0,001 & 0,235 \\
Consumo de energia (kcal/dia) & 0,000 & 0,324 \\
Idade (anos) & 0,463 & 0,014 \\
\hline
\end{tabular}

$r^{2}$ ajustado para as meninas $=0,09$.

AF - Atividadefísica.

\section{Discussão}

Uma das formas de diagnosticar e estudar o sobrepeso, a obesidade e a desnutrição é utilizar 0 IM C, o qual tem sido apontado como um bom indicador desses distúrbios nutricionais em adolescentes ${ }^{1}$.

Sabe-sequeambosos valores elevados delMC, ou seja, que caracterizam sobrepeso ou obesidade, e os valores baixos de IM C, os quais indicam desnutrição, estão associados a várias complicações orgânicas 7,12 . Para sugerir ações que combatam esses distúrbios nutricionais, é fundamental identificar os fatores determinantes do IM C.

O nível de atividade física é considerado um fator que influencia o estado nutricional dos indivíduos ${ }^{13}$. Dowda et al. ${ }^{14}$ avaliaram 2791 jovens entre oito e dezesseis anos de idade envolvidos no Third National Health and Nutrition Examination Survey e verificaram que tanto os meninos quanto as meninas que participavam deprogramas de exercícios físicos e equipes esportivas apresentavam menor chance deter um I M C igual ou maior ao 85 o percentil (sobrepeso) quando comparados com os jovens que não participavam destas atividades (meninos: $O R=0,63$; IC: $0,43-0,91$; meninas: $O R=0,23 ; I C: 0,12-0,44)$.

Levin et al. ${ }^{15}$ relatam que adolescentes com valores de IMC elevados (acima do adequado para a saúde) são menos envolvidos com a prática de atividades físicas do que os adolescentes com valores de IM C mais baixo.

No presente estudo, no entanto, as meninas insuficientemente ativas apresentaram uma mé dia de IMC significativamente maior do que a média das ativas, enquanto que, para os meninos não houve diferença estatística.

Resultado semelhante pode ser observado no estudo de Fonseca et al. ${ }^{16}$ real izado em uma escola particular no município de Niterói (RJ), com 391 adolescentes, de ambos os sexos, com idade entre quinze edezessete anos. Os autores não ob- servaram diferença $(p>0,40)$ entreo nível deatividade física de adolescentes com IM C maior ou igual ao $90^{\circ}$ percentil eo nível deatividadefísica de adolescentes que apresentaram o IM C menor do que o ponto de corte sugerido. Este achado écorroborado pelo estudo de M anios et al. ${ }^{17}$.

Uma possível explicação para estes resultados é que exista um viés de informação, pois os adolescentes com valores de IM C elevados (excesso de peso) podem superestimar seu nível de atividade física, apresentando assim um nível de atividade maior ou igual ao dos indivíduos com valores de IM C mais baixos (eutróficos) ${ }^{18}$. Além disso, pode-sedizer queo IM C não dependeapenas da atividade física, mas também de outros fatores, como a alimentação, ou seja, mesmo tendo um expressivo gasto energético por meio da atividade física, se 0 individuo consumir mais energia do que gasta, poderá engordar ${ }^{13}$.

No presenteestudo, a variável tempo de atividade física semanal não apresentou correlação com o I M C ( $r=0,09 ; p=0,28)$ para os meninos. Para as meninas, essa variável apresentou correlação positiva ( $r=0,22 ; p<0,003)$; porém, ao incluí-la no modelo deregressão, percebeu-seque a mesma perdeu a significância na presença das variáveis idade, maturação sexual e consumo de energia.

Em concordância com o presente trabalho, estudos realizados por Fonseca et al. ${ }^{16} \mathrm{e} \mathrm{M}$ onteiro et al. ${ }^{18}$ constataram que 0 nível de atividade física não se associou com o IM C de adolescentes em ambos os sexos. É oportuno relatar quea coleta de informações sobre atividade física foi contemporânea à do IM C nos dois estudos.

Além do viés de informação, já citado anteriormente, uma possível explicação para estes resultados é que em estudos transversais possa ocorrer a causalidade reversa, ou seja, devido à preocupação com o excesso de peso corporal, os adolescentes com maior IM C procuram fazer mais atividade física para emagrecer, apresentan- 
do no período de coleta de dados o mesmo nível de atividade física daquel es que têm menores valores de IM C 16,18 .

No presente trabalho, observou-se alta prevalência de adolescentes insuficientementeativos e meninos significativamente mais ativos do que as meninas. Outros estudos também mostraram elevada prevalência de adolescentes insuficientemente ativos e o maior nível de atividade física por parte dos meninos ${ }^{19,20}$.

Deacordo com M anios et al. ${ }^{21}$, essa diferença denível deatividadefísica entreos sexos não deve ser atribuída às diferenças sexuais fisiológicas (quantidade de massa muscular, débito cardíaco etc.), mas às crenças culturais e sociais de pais e professores que estimulam mais os meninos do que as meninas a praticarem esportes eatividade física em geral.

Em relação ao consumo de energia dos adolescentes do presentetrabal ho, identificou-seque o mesmo é consumido em quantidades muito próximas quando comparado entre os sexos ( meninos = 3.408,7 kcal; meninas 3.405,3 kcal; p $>0,05)$. Garcia et al..$^{22}$, ao avaliarem o consumo de energia de adolescentes com idade entre dez e catorze anos de um centro de juventude do município de São Paulo, também não encontraram diferença $(p=0,90)$ entre as médias de consumo de energia de meninos e meninas (2.026,1 kcal; $1.842,7 \mathrm{kcal}$, respectivamente).

$\mathrm{N}$ a presente pesquisa, não foi identificada correlação significativa entre o IM C e o consumo de energia, tanto para os meninos quanto para as meninas. Da mesma forma, em estudo realizado por $\mathrm{M}$ anios et al. ${ }^{17}$ envolvendo adolescentes com idade entre doze e treze anos, observou-se que, para ambos os sexos, não houve associação significativa entre o consumo de energia e o IMC. Outros estudos corroboram os achados anteriormente citados ${ }^{16,23}$. Mais uma vez, uma provável explicação para esse resultado está relacionada à limitação de estudos transversais a exemplo deste, pois adolescentes com maiores valores de IMC podem restringir a ingestão de alimentos com o objetivo de emagrecer, informando no momento da coleta um consumo de energia reduzido ${ }^{16}$. Outra possibilidade éque os adolescentes com valores de I M C elevados (obesos) costumam subestimar o consumo de alimentos, apresentando valores de consumo de energia parecidos com os adolescentes com valores de IMC adequados para saúde (eutróficos) ${ }^{18,23}$.

Pôde-se observar, neste trabalho, que apesar da média de idade dos adolescentes de ambos os sexos ser muito próxima, constatou-se maior nú- mero de meninos pré-púberes ( meninos $=34 \%$; meninas $=10 \% ; p<0,001$ ). Provavelmente devido ao fato de que, em geral, as meninas iniciam a fase de maturação sexual antes que os meninos ${ }^{8}$.

Tanto os meninos quanto as meninas púberes do presente estudo apresentaram uma média de IM C significativamente maior quando comparada com a média de IMC dos pré-púberes. Além disso, independente dos minutos de atividadefísica, consumo alimentar eidade, existeuma associação positiva e significativa entre IMC e maturação sexual.

Resultados similares foram encontrados por Coelho et al. ${ }^{24}$ ao avaliarem o IMC e estágio puberal de 466 adolescentes com idade entre dez $\mathrm{e}$ vinte anos (RJ). Os autores verificaram uma associação positiva e significativa entre I M C e estágio puberal para ambos os sexos.

0 estudo de Wang ${ }^{25}$, com adolescentes entre oito e catorze anos de idade, apresenta achados semel hantes ao do presenteestudo para as meninas, porém são diferentes dos resultados encontrados para os meninos. Constatou-se que a maturação sexual precoce apresentou associação positiva e estatisticamente significativa com - IMC para as meninas, enquanto que para os meninos ela foi negativa e não significativa.

Em relação às meninas, esses resultados são esperados, poisa maturação sexual contribui para o ganho do peso corporal devido à maior atuação do estrógeno, o qual estimula 0 aumento dos depósitos de gordura 8 .

Quanto aos resultados sobre a relação maturação sexual eIM C em meninos, pode-se afirmar que estudos sobre esse assunto são relativamente escassos e muitas vezes controversos. Uma provável explicação para esses resultados é que osmeninos geralmenteaumentam principalmente a massa muscular e a massa óssea durante a maturação sexual, podendo muitas vezes reduzir a quantidade de gordura corporal durante essa fase ${ }^{8}$. Dessa forma, enquanto alguns meninos aumentam o IMC devido ao aumento da massa magra, outros, apesar do ganho de massa magra, mantêm ou reduzem o IMC devido à perda de gordura corporal.

No queserefereàinfluência da idadeno I M C das meninas, verificou-se que as adolescentes de catorze e quinze anos de idade apresentaram a média de IM C estatisticamente maior do que a média de IM C das adolescentes de dez anos. Verificou-se, também, que à medida que aumenta a idade, cresce o I M C, mesmo quando esta variável é incluída no modelo de regressão múltipla. Já para os meninos, a variável idade não foi esta- 
tisticamente relacionada ao IMC e não foram encontradas diferenças estatisticamente significativas entre as médias do IM C para as categorias de idade desse grupo.

Diferente dos resultados do presente estudo, Fonseca et al. ${ }^{16}$ observaram que o IMC dos me ninos aumentava deacordo com a idade, enquanto que para as meninas não foi identificada associação entre IM C eidade, provavelmenteporque as mesmas se encontravam em faixa etária de crescimento reduzido; além disso, o grupo de meninas avaliadas nesse estudo demonstrava grande preocupação com o "manter-se magra".

Dessa forma, pode-se concluir que a maturação sexual é determinante do IMC, para ambos os sexos, reforçando a idéia de que é necessária a avaliação da maturação sexual em estudos que tenham como objetivo avaliar o estado nutricional ou analisar fatores que possam influenciar o mesmo. Verifica-se também que a idade foi fator determinante do IMC para as meninas, conhecimento esse que deve ser considerado pelos profissionais da área da saúde com o objetivo de ori- entar as adolescentes sobre a importância de se prevenir contraa obesidadenessafase, assim como auxiliá-las a compreender as transformações que inevitavelmente ocorrerão com o próprio corpo.

É importante ressaltar que tanto o delineamento do estudo (transversal) quanto os métodos utilizados não foram eficientes para avaliar o efei to da atividadefísica edo consumo alimentar no IMC. Sugere-se para próximos estudos um delineamento mais específico para avaliar causa e efeito, assim como o aprimoramento dos instrumentos utilizados para avaliar nível de atividade física e consumo alimentar.

Entende-se que o aperfeiçoamento dos mé todos para este tipo de estudo é de extrema relevância, pois a partir do conhecimento dos fatores determinantes do IMC, é possível formular estratégias de orientações que proporcionem aos adolescentes o desenvolvimento de um estilo de vida ativo e saudável, sendo que na adolescência o ser humano ainda tem facilidade para adquirir hábitos saudáveis que deverão ser mantidos por toda a vida.

\section{Colaboradores}

A Romero e B Slater trabalharam em todas as etapas da elaboração do artigo; AA Florindo e M RD O Latorretrabalharam na metodologia, nas análises estatísticas e na discussão; M V Silva e C Cezar trabalharam na introdução, discussão e revisão crítica. 


\section{Referências}

1. World Health Organization. Physical status: the use and interpretation of anthropometry [Technical Report Series, 854]. Geneva: WH O; 1995.

2. Cintra IP, Costa RF, Fisberg M. Composição corporal na infância e adolescência. In: Fisberg $M$ organizador. Atualização em obesidade na infância e adolescência. São Paulo: Atheneu; 2005. p. 33-46.

3. Organização Mundial da Saúde. O besidade: prevenindo e controlando a epidemia global. São Paulo: Roca; 2004.

4. Wang Y, M onteiro C, Popkin BM. Trends of obesity and underweight in older children and adolescents in the United States, Brazil, China, and Russia. Am J Clin Nutr 2002; 75(6):971-977.

5. Andrade RG, Pereira RA, Sichieri R. Consumo alimentar de adolescentes com e sem sobrepeso do município do Rio de Janeiro. Cad Saude Pyblica 2003; 19(5):1485-1495.

6. Patrick K, Norman GJ, Calfas KJ, Sallis JF, Zabinski MF, Rupp J, Cella J. Diet, physical activity, and sedentary behaviors as risk factors for overweight in adolescence. Arch Pediatr Adolesc M ed 2004; 158(4):385-390.

7. Kurpad AV, Muthayya S, Vaz M. Consequences of inadequate food energy and negative energy balance in humans. Public Health Nutr 2005; 8(7 Supl):1053-1076.

8. Rogol AD, Roemmich JN, Clark PA. Growth at puberty. J Adolesc Health 2002; 31(6 Supl):192-200.

9. Florindo AA, Romero A, Peres SV, Silva MV, Slater B. Desenvolvimento, validação e reprodutibilidade de um questionário para avaliação da atividade física em adolescentes. Rev. Saude Publica 2006; 40(4):802-809.

10. Pate PR, Freedson PS, Sallis JF, Taylor WC, Sirard J, Trost SG, Dowda M. Compliance with physical activity-guidelines: prevalence in a population of children and youth. Ann Epidemiol 2002; 12(5):303-308.

11. Slater B, Philippi ST, Fisberg RM, Latorre MR. Validation of a semi-quantitative adolescent food frequency questionnaire applied at a public school in São Paulo, Brazil. Eur J Clin Nutr 2003; 57(5):629635.

12. Daniels SR, Arnett DK, Eckel RH, Gidding SS, Hayman LL, Kumanyika S, Robinson TN, Scott BJ, St Jeor S, Williams CL. Overweight in children and adolescents: pathophysiology, consequences, prevention, and treatment. Circulation 2005; 111(15):1999-2012.

13. Bouchard C. Atividade física e obesidade. São Paulo: Manole; 2003. p.3-22.

14. Dowda M, Ainsworth BE, Addy CL, Saunders R, Roner W. Environmental influences, physical activity and weight status in 8- to 18-year-olds. Arch Pediatr Adolesc M ed 2001; 155(6):711-717.
15. Levin S, Lowry R, Brown DR, Dietz WH. Physical Activity and Body Mass Index Among US Adolescents: youth risk behanior survey, 1999. Arch Pediatr Adolesc M ed 2003; 157(8):816-820.

16. Fonseca VM, Sichieri R, Veiga GV. Fatores associados à obesidade em adolescentes. Rev. Saude Publica 1998; 32(6):541-549.

17. Manios $Y$, Kolotourou $M, M$ oschonis $G$, Sur $H$, Keskin Y, Kocaoglu B, Hayran O. Macronutrient, physical activity, serum lipids and increased body weight in primary schoolchidren in Instanbul. Pediatr Int 2005; 47(2):159-166.

18. Monteiro P, Victora C, Barros F. Fatores de risco sociais, familiares e comportamentais para obesidade em adolescentes. Rev Panam Salud Publ/Pan Am J Public Health 2004; 16(4):250-2558.

19. Farias Junior JC, Lopes AS. Comportamentos de risco relacionados à saúde em adolescentes. Rev Bras Ci M ov 2004; 12(1):7-12.

20. Hallal PC, Wells JC, Reichert FF, Anselmi L, Victora CG. Early determinants of physical activity in adolescents prospective birth cohort study. BM J 2006; 332(7548):1002-1007.

21. Manios $Y$, Kafatos A, Codrington C. Gender differences in physical activity and physical fitness in young children in Crete. J Sports M ed Phys Fitness 1999; 39(1):24-30.

22. Garcia GCB, Gambardella AMD, Frutuoso MFP. Estado nutricional e consumo alimentar de adolescentes de um centro de juventude da cidade de São Paulo. Rev. Nutr. 2003; 16(1):41-50.

23. Ortega RM, Requejo AM, Andrés P, Lopes-Sobaler $A M$, Redondo R, Gonzalés-Fernadez M. Relationsship between diet composition and body mass index in a group of Spanish adolescents. Br J Nutr 1995; 74(6):765-773.

24. Coelho KSC, Sichieri R, González HC. Influencia de la maduracion sexual em el índice de massa corporal em adolescentes de bajo nível socioeconômico de Rio de Janeiro. Rev Chil Nutr 2002; 29(1):33-39.

25. Wang Y. Is obesity associated with early sexual maturation? A comparison the association in American boys versus girls. Pediatrics 2002b; 110(5):903-910.

Artigo apresentado em 26/02/2008

Aprovado em 05/05/2008

Versão final apresentada em 16/07/2008 